\title{
Endobronchialklappen: Eine Alternative für bestimmte Patientengruppen?
}

Die chirurgische Resektion embolischer Lungensegmente ist mit hohen Komplikationsraten assoziiert. Eine neue, weniger invasive Behandlungsmöglichkeit ist die Implantation von Endobronchialklappen, die sich nur in eine Richtung öffnen: Sie sorgen für die Entlüftung der überblähten Areale und verhindern die Wiederbelüftung. Ihr Therapieerfolg ist bei Patienten ohne Kollateralatmung besonders ausgeprägt, dies zeigt eine aktuelle Studie.

N Engl J Med 2015; 373: 2325-2335

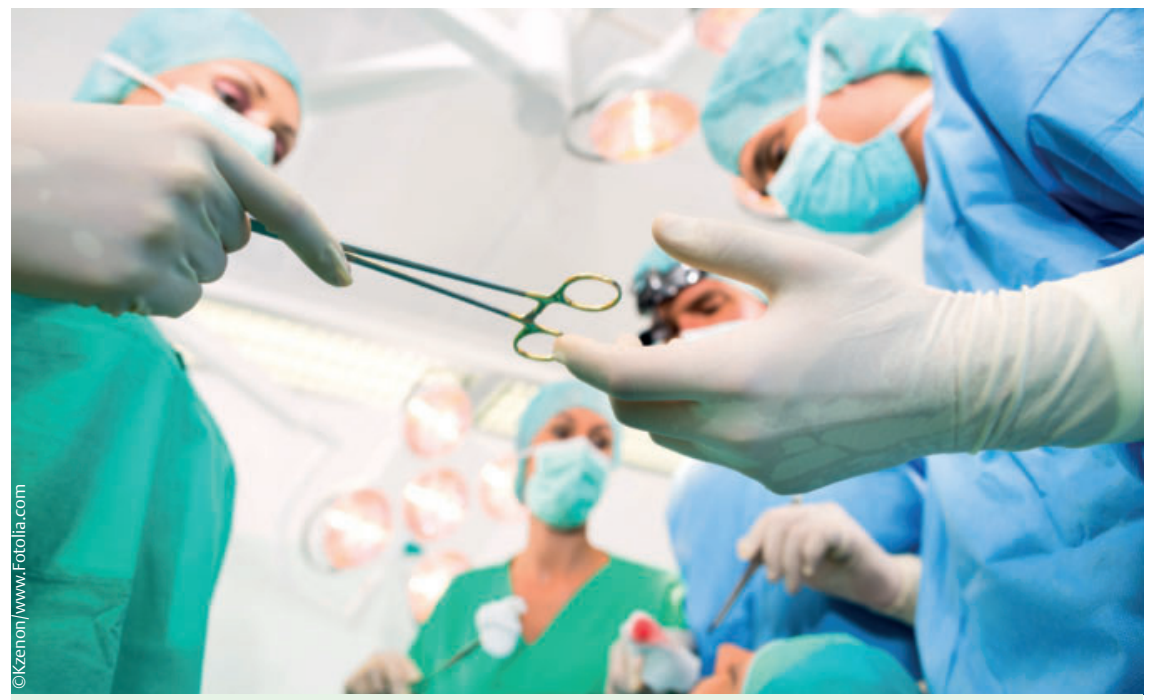

Die endoskopische Lungenvolumenreduktion bietet die Option zur Verbesserung der Lebensqualität selektierter Patienten mit fortgeschrittenem Lungenemphysem. Hierbei stellt die Implantation von Endobronchialklappen (auch als Ventile bezeichnet) ein reversibles Verfahren dar.

Frühere Studiendaten weisen darauf hin, dass Patienten, die in der High Resolution Computertomografie (HRCT) eine intakte Bindegewebswand zwischen geschädigtem Lobulus und Nachbararealen zeigen und bei denen auch sonst nichts für eine Kollateralatmung spricht, von der Implantation der Bronchialklappen besonders profitieren.

Um dies zu überprüfen, untersuchten K. Klooster et al. aus den Niederlanden in einer offenen, randomisierten, kontrollierten Studie 84 Patienten mit schwerem Emphysem, deren HRCT-Bildgebung eine vollständige Abgrenzung der geschädigten Lungensegmente zeigte. 13 Patienten wurden ausgeschlossen, nachdem in der Bronchoskopie wider Erwar- ten doch noch eine Kollateralatmung sichtbar wurde. Bei 3 Patienten waren die betroffenen Bronchiolen nicht erreichbar. Die übrigen 68 Patienten erhielten entweder Endobronchialklappen, die wie einseitige Ventile wirken (Verumgruppe, $n=34$, je 2-7 Klappen) oder sie bekamen zunächst nur eine medikamentöse Standardbehandlung (Kontroll- bzw. Wartegruppe, $n=34)$. Zu Studienbeginn, nach einem und nach 6 Monaten wurden das forcierte Exspirationsvolumen in einer Sekunde $\left(\mathrm{FEV}_{1}\right)$ und die forcierte $\mathrm{Vi}$ talkapazität (FVC) ermittelt und ein 6-Minuten-Gehtest durchgeführt. Die Änderung dieser Parameter bis Monat 6 war das primäre Studienziel. Anschließend wurden auch die Kontrollpatienten mit Endobronchialklappen versorgt.

\section{Besseres Outcome bei primären Endpunkten \\ $\nabla$}

Tatsächlich übertrafen die Verum- nach 6 Monaten die Kontrollpatienten:

- um $140 \mathrm{ml}$ beim $\mathrm{FEV}_{1}$-Anstieg,

- um $347 \mathrm{ml}$ beim FVC-Anstieg und

- um 74 m beim 6-Minuten-Gehtest.

Der Vorteil war jeweils statistisch signifikant. Auch in den sekundären Studienendpunkten waren die „Klappenpatienten“ erfolgreicher. Bei den ursprünglichen Kontrollpatienten zeigten sich nach der Implantation ähnliche Fortschritte.

\section{Pneumothorax und erneute Bronchoskopie}

$\nabla$

Die Besserung der primären Endpunkte wurde erkauft durch eine höhere Rate unerwünschter Ereignisse. So erlitten 6 der 34 Klappenpatienten (18\%) einen Pneumothorax, bei einem von ihnen mussten die Ventile zeitweilig und bei 2 weiteren dauerhaft entfernt werden.

Insgesamt hatten 7 von 34 Verumpatienten $(21 \%)$ inakzeptable Nebenwirkungen, die eine Entfernung der Endobronchialventile notwendig machten. Bei 12 von 34 Klappenempfängern (35\%) musste aus verschiedenen Gründen eine erneute Bronchoskopie durchgeführt werden. Die Verträglichkeit der Klappenimplantation war jedoch immerhin besser als die bisher beobachtete Verträglichkeit der einzigen Alternative, der chirurgischen Segmentresektion, merkten die Autoren an.

\section{Fazit}

Bei Patienten mit schwerem Emphysem und nachweislichem Fehlen von Kollateralatmung besserte die Endobronchialklappenimplantation die Lungenfunktion und körperliche Belastbarkeit. Es traten schwere, teils lebensbedrohliche unerwünschte Wirkungen auf, diese erforderten eine sorgfältige Nachbeobachtung und -behandlung, so die Autoren.

\section{Simone Reisdorf, Erfurt}

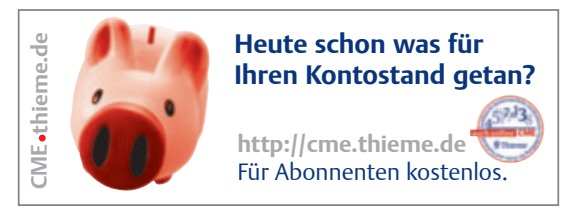

